\title{
Flow Stress and Work-Hardening of Pearlitic Steel
}

\author{
By Toshihiko Takahashi* and Michihiko Nagumo*
}

\begin{abstract}
The mechanism which dominates the flow stress and work-hardening of pearlitic steel at small strains is investigated from both the analysis of tensile properties and microscopic observations of slip patterns. The flow stress and the work-hardening rate are shown to increase linearly with an inverse square root of the mean free ferrite path. The flow behavior of pearlite is characterized by the increase in the Petch slope with strain and by the minor dependence of the flow stress and work-hardening on the strain rate. Analysis of a strain-rate cycling experiment shows that a large part of the flow stress is the long-range internal stress and that the effective stress acting on dislocations is almost the same as that in ferritic iron. Microscopic observations have revealed that the increase in the density of dislocations takes place preferentially along the ferrite-cementite interface and that slip bands extend along the grain boundaries and through the gaps of lamellar cementites. Shear of cementites is eventually observed at the intersections with slip bands. Such features of the deformation of pearlite are consistently explained by the model that dislocations, which are generated at the interface between cementite and ferrite rather than those multiplied within the ferrite grains, develop a large amount of long range internal stress within the pearlitic ferrite. Deformation of comentites is not likely to affect the flow stress or work-hardening of pearlite.
\end{abstract}

(Received October 31, 1969)

\section{Introduction}

Deformation of pearlite has long been a subject of many investigations on the mechanical properties of steels. Studies on the strengthening mechanism, however, have not been made until rather recent years. Comprehensive studies by Gensamer et al. ${ }^{(1)(2)}$ on the effect of the cementite dispersion upon the strength of carbon steels incorporating with the results by succeeding investigations ${ }^{(3)}$ (5), have lead to the understanding that strengthening by the dispersion hardening mechanism ${ }^{(6)}$ is of primary importance. Recent microscopic observations of pearlitic steels subjected to large deformation have revealed that subgrains are formed within the pearlitic ferrite ${ }^{(7)(8)}$. The flow stress of the deformed pearlitic steel was shown to be governed by the subgrain size which is reduced in dimensions with the increase in the amount of strain and with the decrease in the original interlamellar spacing. In this respect, lamellar cementites play a secondary role in the flow stress through the subgrain size. The dependence of the flow stress on the interlamellar spacing or on the subgrain size was represented by a modified HallPetch relationship where the Petch slope increases with strain ${ }^{(7)}$. However, no satisfactory explanations have been made of the significance of the modified Hall-Petch relationship.

From optical and electron microscopic observations,

* Tokyo Research Institute, Yawata Iron \& Steel Co., Ltd., Kawasaki, Japan.

(1) M. Gensamer, E. B. Pearsall and G. V. Smith : Trans. ASM, 28 (1942), 380.

(2) M. Gensamer, E. B. Pearsall, W. Pellini and J. R. Low, Jr. : ibid, 28 (1942), 983.

(3) C. S. Robert, R. C. Carruthers and B. L. Averbach : ibid, 44 (1952), 1150.

(4) A. M. Turkalo and J. R. Low, Jr. : Trans. Met. Soc. AIME, $212(1959), 750$.

(5) W. R. Tyson : Acta Met., 11 (1961), 61.

(6) E. Orowan : Discussion, Symposiam on Internal Stresses, Inst. Metals, London, (1947), p. 451.

(7) J. D. Embury and R. M. Fisher : Acta Met., 14 (1966), 147.

(8) M. A. P. Dewey and G. W. Briers : J. Iron Steel Inst., 204 (1966), 102. many experimental evidences have been presented on the deformation of lamellar cementite during mechanical working processes ${ }^{(9) \sim(12)}$. It should be worth noticing that slip in the pearlitic ferrite occasionally runs traversing across lamellar cementites at their growth faults ${ }^{(10)}$ This might suggest the possibility that the structure of cementite affects the flow stress of pearlitic steel. Another important observation of deformed pearlitic steel using an electron microscope is that the interface between ferrite and cementite acts as the nucleation site of dislocations ${ }^{(13)}$. Such a role of the interface is a noticeable characteristic of the pearlitic steel, being quite different from the grain boundary in ferritic iron.

A recent analysis of the flow stress of the pearlitic and spheroidized steels showed that the activation energy and activation volume of the movement of dislocations are almost identical with those of ferritic iron ${ }^{(14)}$. From this analysis, cementites are considered to be immaterial to the thermal process of the movement of dislocations.

Thus, in spite of many studies from different aspects, any consistent model has never been proposed to relate the microscopic observations with the flow behavior of pearlitic steel. The present investigation was undertaken with the aim to reveal the governing mechanism of the deformation of pearlitic steel, connecting the analysis of the flow stress with the deformation patterns exhibited by microscopic observations.

\section{Experimentals}

A plain carbon steel prepared from electrolytic iron was melted in a vacuum induction furnace and cast to a $20 \mathrm{~kg}$ ingot. The chemical composition is given in Table 1.

(9) J. C. Danko and R. D. Stout : Weld. Journ. Suppl, 34 (1955), 113-S.

(10) K. E. Puttick : J. Iron Steol Inst., 185 (1956), 167.

(11) A. S. Keh : Acta Met., 11 (1963), 1101.

(12) R. M. Fisher : Electron Microscopy 1966, Maruzen, Tokyo, (1966), p. 425.

(13) F. B. Pickering : Iron \& Steel, 38 (1965), 110.

(14) S. Karashima and T. Sakuma : Proc. 11 th Japan Congress on Material Research, The Soc. Material Science, Japan, 1968. 
After hot-rolling the ingot to the thickness of $12 \mathrm{~mm}$, tensile specimens with a gauge diameter of $6 \mathrm{~mm}$ and a gauge length of $48 \mathrm{~mm}$ were prepared. Samples with different interlamellar spacings were produced by austenitizing at $1000^{\circ} \mathrm{C}$ followed by isothermal transformation into pearlite at various temperatures between $700^{\circ}$ and $550^{\circ} \mathrm{C}$. For microscopic observations, the surface of a flat specimen with gauge dimensions of $6 \mathrm{~mm}$ in width, $20 \mathrm{~mm}$ in length and $1 \mathrm{~mm}$ in thicktress was polished and etched by picral before the tensile test.

Table 1 Chemical composition of the specimens

\begin{tabular}{l|l|l|l|l|l|l}
\hline \hline $\mathrm{C} \%$ & $\mathrm{Si} \%$ & $\mathrm{Mn} \%$ & $\mathrm{P} \%$ & $\mathrm{~S} \%$ & $\mathrm{O} \%$ & $\mathrm{~N} \%$ \\
\hline 0.79 & 0.02 & 0.02 & 0.003 & 0.003 & 0.003 & 0.0012 \\
\hline
\end{tabular}

The degree of refinement of the pearlite is expressed in this paper by the "mean free ferrite path" (mffp) which denjtes the mean uninterrupted path through the ferrite. The mffp of each specimen was determined as the mean distance between cementites along lines drawn on electron micrographs with many pearlite colonies. In order to average the orientations of lamellar cementites with the tensile direction, a set of two lines perpendicular to each other is drawn arbitrarily on every twenty-four micrographs. The number of counts of the free ferrite path on a photograph is about forty on the average.

Tensile tests were conducted at room temperature using an Instron type machine at strain rates between $2 \times 10^{-5}$ and $5 \times 10^{-2}$ per second. In order to distinguish the faults of lamellar cementite due to the deformation from those initially present, microscopic observations were carried out with two stage cellulose acetate-carbon replicas of the same area of a specimen surface before and after the tensile test. This technique was useful in revealing the correspondence between the cementite morphology and the mode of deformation. Transmission electron microscopy was also carried out to study the early stage of deformation.

\section{Experimental Results}

\section{Dependence of the flow stress on the pearlite morphology}

It is a well established fact that the flow stress of pearlite increases with the refinement of pearlite lamellae $^{(1)(2)}$. A result of the present study is shown in Fig. 1 , where the flow stress is plotted against the inverse squre root of mffp. The flow stress can be related to mffp by the Hall-Petch relationship ${ }^{(15)(16)}$. It is of interest to note that this relationship in the case of pearlite is characterized by the increase in the Petch slope with strain, as is shown in Fig. 2 for various strain rates. This trend is qualitatively consistent with the result by Embury and Fisher ${ }^{(7)}$ but quantitatively in disagreement as will be discussed in the next section.

The increase in the flow stress is also an indication of

(15) E. O. Hall : Proc. Phys. Soc., B 64 (1951), 747.

(16) N. J. Petch : J. Iron Steel Inst., $174(1953), 25$. the increase in work-hardening. Although workhardening rate, defined as the tangent of stress-strain curve, has some inevitable experimental errors in the numerical value, its dependence on $\mathrm{mffp}$ is represented

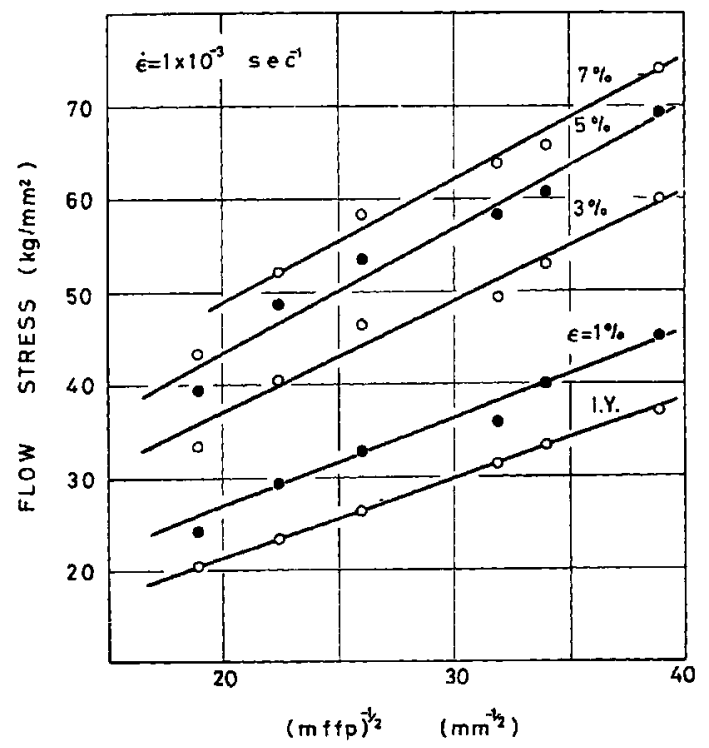

Fig. 1 Dependence of the flow stress on the mean free ferrite path at a strain rate of $1 \times 10^{-3}$

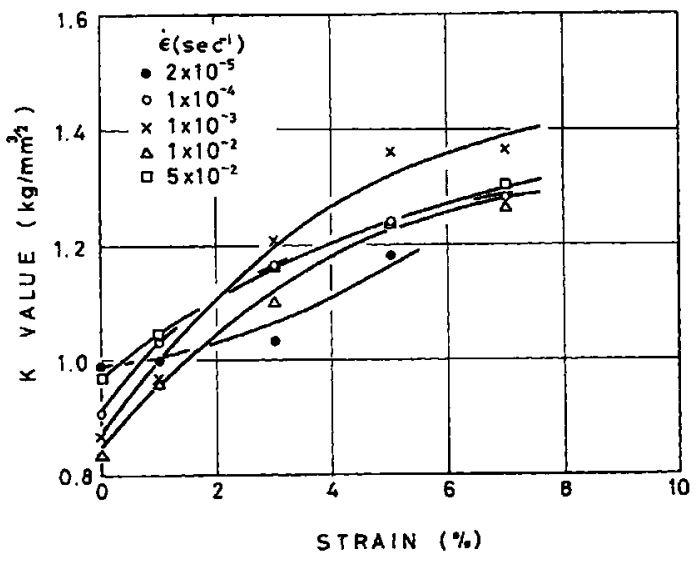

Fig. 2 Strain dependence of the Petch slope at various strain rates

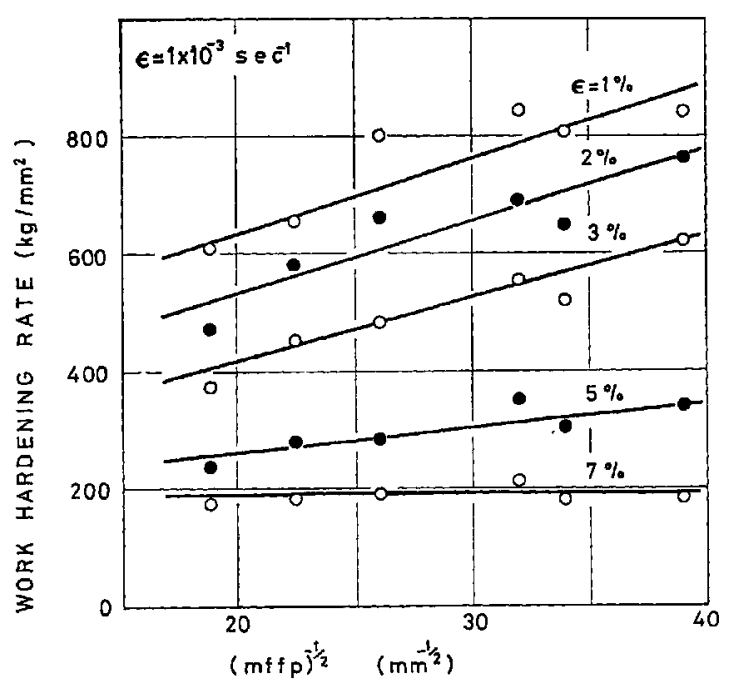

Fig. 3 Dependence of the work-hardening rate on the free ferrite path of the specimen at strain rate of mean $1 \times 10^{-3} \mathrm{sec}^{-1}$ 
fairly well by a linear function of the inverse square root of mffp. The absolute value of the work-hardening rate and its dependence on the $\mathrm{mff} p$ are reduced at larger strains. These are shown in Fig.3.

\section{Dependence of the flow stress on the strain rate}

In contrast with their strong dependence on both the strain rate and temperature in ferritic iron, changes of the flow stress and work-hardening rate with the strain rate in the case of pearlite are rather small fractions as shown in Fig. 4 and Fig. 5. An example of workhardening of ferritic iron ${ }^{(19)}$ is also graphed in Fig. 5 for comparison.

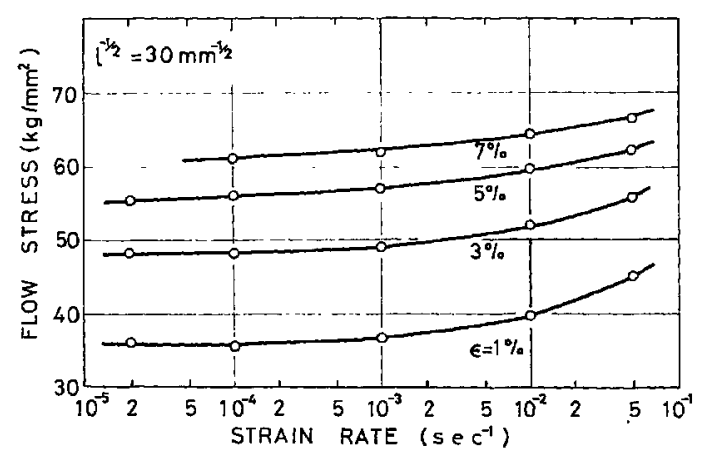

Fig. 4 Strain-rate dependence of the flow stress of the specimen with $\mathrm{mff} p 1.1$ micron

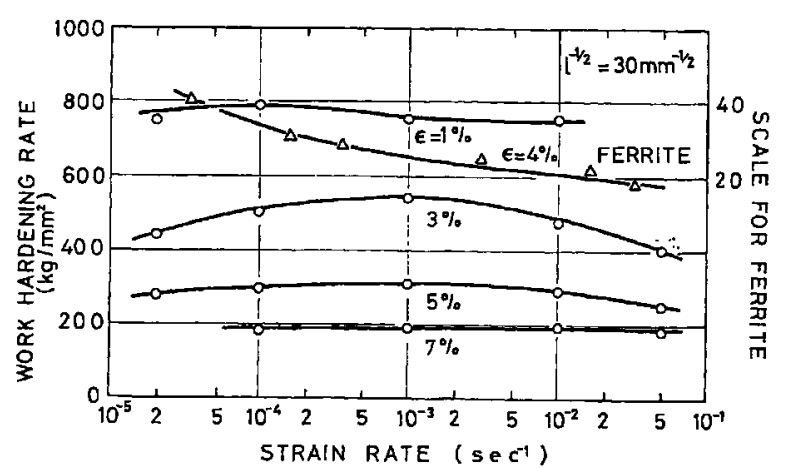

Fig. 5 Strain-rate dependence of the work-hardening rate of the specimen with mffp 1.1 micron. The data of ferritic iron is from Ref. (19)

\section{Evaluation of the effective stress acting on dislocations}

The strengthening mechanism of a material is likely inferred from the parameters which characterize the movement of dislocations. Incorporating with the activation energy and activation volume of pearlite which were obtained previously by Karashima et al. ${ }^{(14)}$, the evaluation of another important parameter, the effective stress, is attempted in this study. The effective shear stress $\tau^{*}$ and the stress exponent $m^{*}$ for the dislocation velocity $v$ are defined by the empirical equation

$$
v=\left(\frac{\tau^{*}}{\tau_{0}}\right) m^{*}
$$

and can be obtained from a strain-rate cycling experiment (17) (19)

(17) R. W. Guard : Acta Met., 9 (1961 $\rangle, 163$.

(18) W. G. Johnson and D. F. Stein : ibid, 11 (1.963), 317.

(19) J. T. Michalak : ibid, 13 (1965), 213.
If it is assumed that the density of mobile dislocations does not change during strain-rate cycling, and that the internal stress vanishes at zero strain, the value of $m^{*}$ is determined by extrapolating to zero strain the values of $m$ at different strains which are given by the equation

$$
m=\Delta \ln \dot{\varepsilon} / \Delta \ln \tau \text {. }
$$

The extrapolation is shown in Fig. 6 and the resulting value of $m^{*}$ is about 20 . This value is identical with that obtained by Karashima and Sakuma ${ }^{(21)}$, but is about four times larger than the value of $m^{*}$ previously obtained $^{(19)(20)}$ with ferritic iron. The cause of the overestimation may be partly due to the internal stress existing even at zero strain. Since the previous assumption implies that the change in the flow stress by the strain-rate cycling is equivalent to the change in the effective stress, the effective stress is estimated in terms of the equation

$$
\left.\tau^{*}=\Delta \tau /\left[\dot{\varepsilon}_{2} / \dot{\varepsilon}_{1}\right)^{1 / m *}-1\right] .
$$

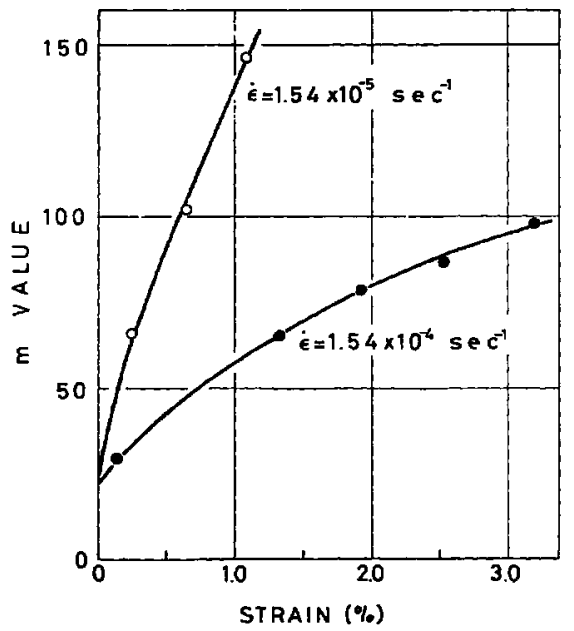

Fig. 6 Strain dependence of the dislocation velocity exponent ' $m$ '

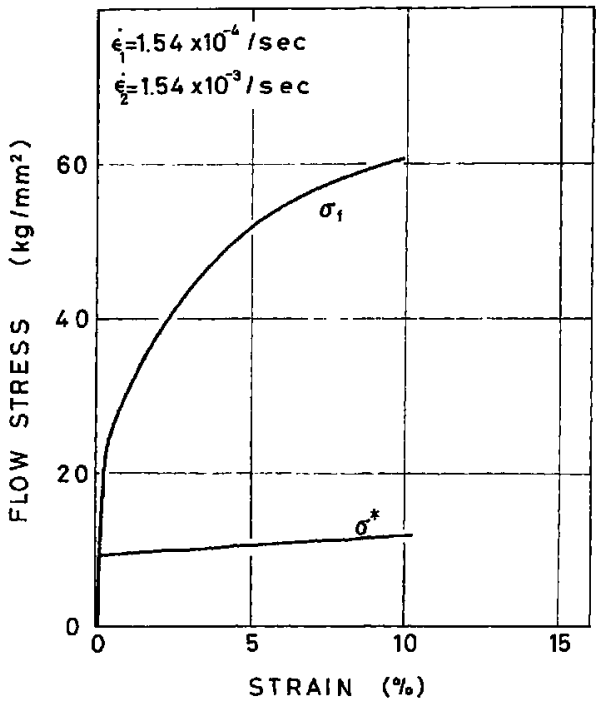

Fig. 7 The ef fective stress $\sigma^{*}$ acting on dislocations in pearlite, caculated from eq.(3) with the $m$-value of 20 . The strain rate is changed from $\dot{\varepsilon}_{1}$ to $\dot{\varepsilon}_{2}$. The flow stress $\sigma_{f}$ is the one at $\dot{\varepsilon}_{1}$

(20) M. Nagumo and H.Matsuda : Proceedings of the International Conference on the Strength of Metals and Alloys, Supplement to Trans. JIM, Vol. 9, (1968), p. 885.

(21) S. Karashima and T. Sakuma : Trans. JM, 9(1968), 63. 
Even though the overestimation of $m^{*}$ should result in the larger value of $\tau^{*}$, the effective stress thus obtained takes only a small part in the entire flow stress as shown in Fig. 7. Taking into account the overestimation of $m^{*}$, the true effective stress is considered to be almost of the same order as that of ferritic iron ${ }^{(19)(20)}$, and the major component of the flow stress is regarded to be the long range internal stress.

\section{Microscopic observations}

The deformation process of the pearlitic steel is characterized by slip patterns. In the early stage of deformation, slip markings on the specimen surface are too fine to be identified, so that they were observed by means of transmission electron microscopy. Photo. 1 (a) (b) are transmission electron micrographs of the specimens undeformed and elongated $2 \%$ respectively. In accor- dance with Pickering ${ }^{(13)}$, the density of dislocations increases preferentially at the interface between ferrite and cementite, suggesting that the interface is an active nucleation site of dislocations.

In an attempt to relate the path of the slip with the cementite morphology, the same area of the specimen surface was examined with replicas before and after the tensile test. For this purpose, a flat specimen, the surface of which is prepared for microscopic observations, was elongated by $7.8 \%$ at a strain rate of $1 \times 10^{-8}$ per second. Large deformations are seen to take place along the grain boundaries (Photo. 2-A) and through the gaps of lamellar cementites (Photo. 2-B, Photo. 3-A).

The slip after sufficient extension eventually gives rise to the transverse shear of cementites (Photo. 2-C, Photo. 3-A). It is worth noticing that the direction of shear is not always coincident with that of maximum shear stress

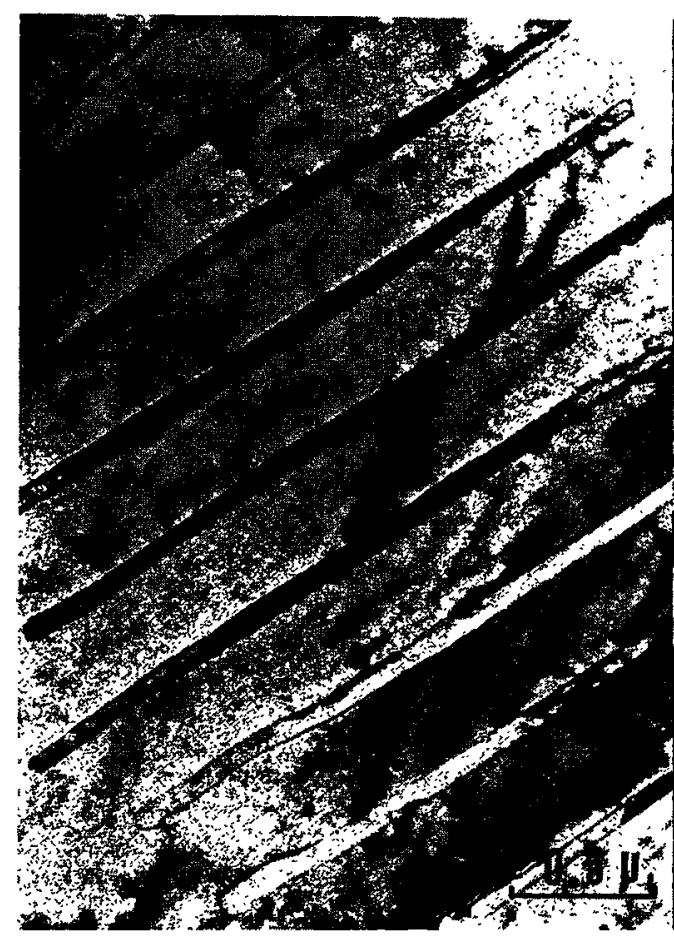

(a) Before deformation

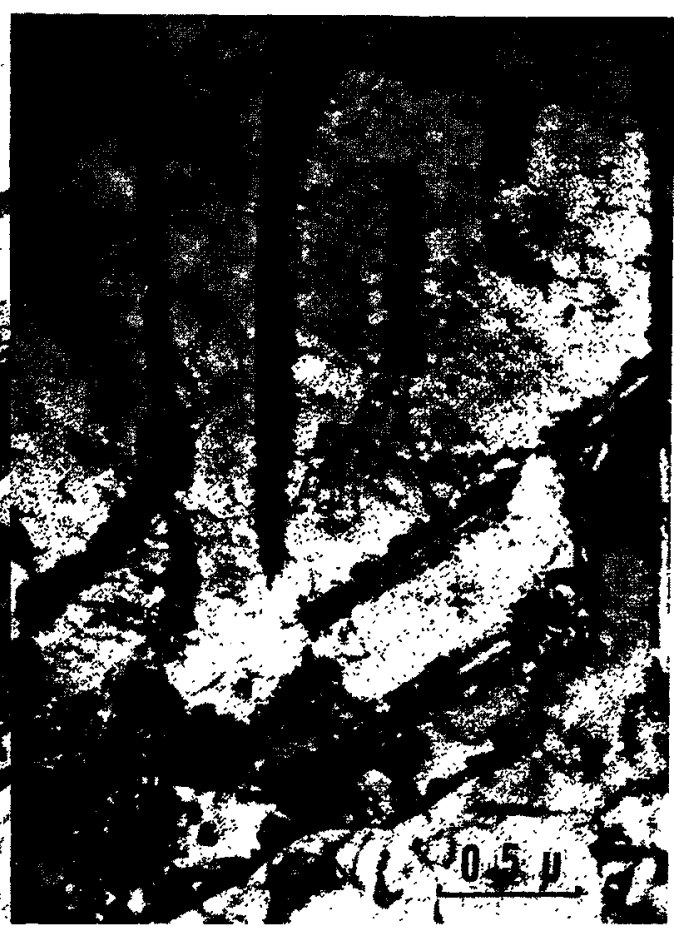

(b) After $2 \%$ extension

Photo. 1 Transmission electron micrograph of specimens

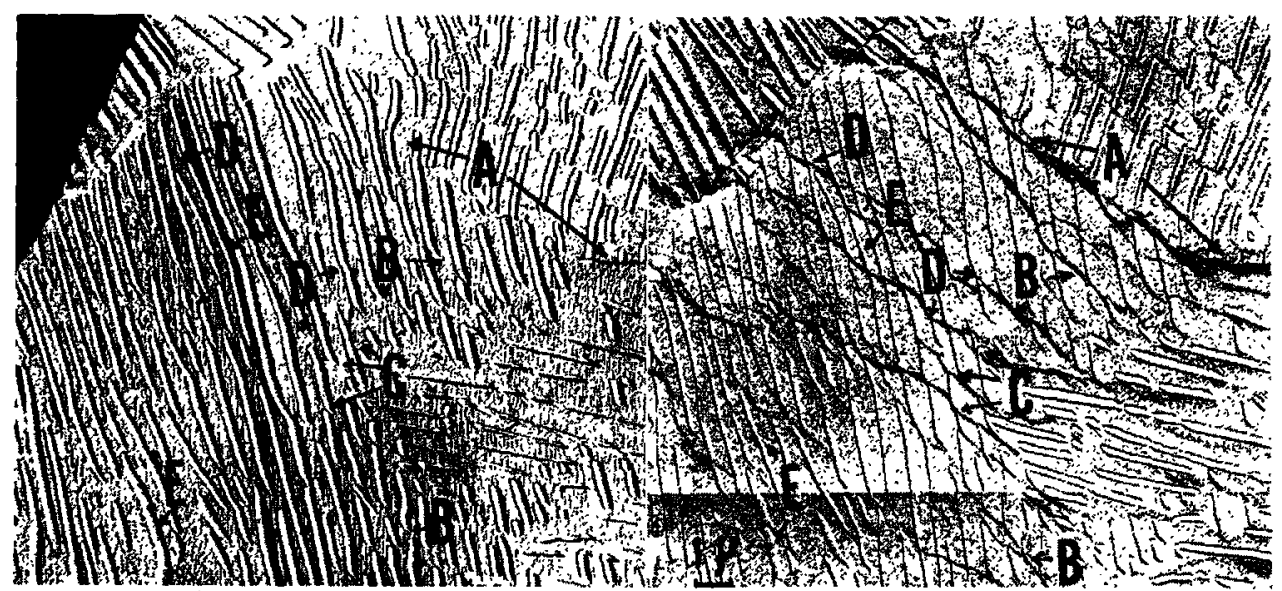

(a) Before tensile test

(b) After 7.8\% extension

Photo. 2 Two stage replication electron micrograph of the surface of a specimen hefore (a),and after (b) $7.8 \%$ extension 


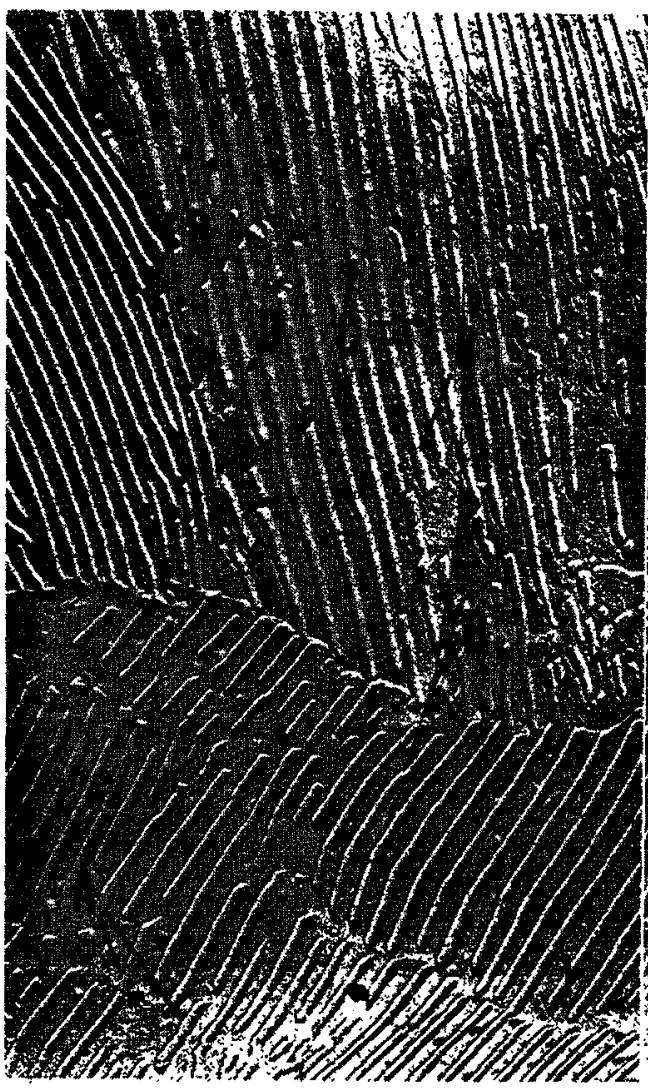

(a) Before deformation

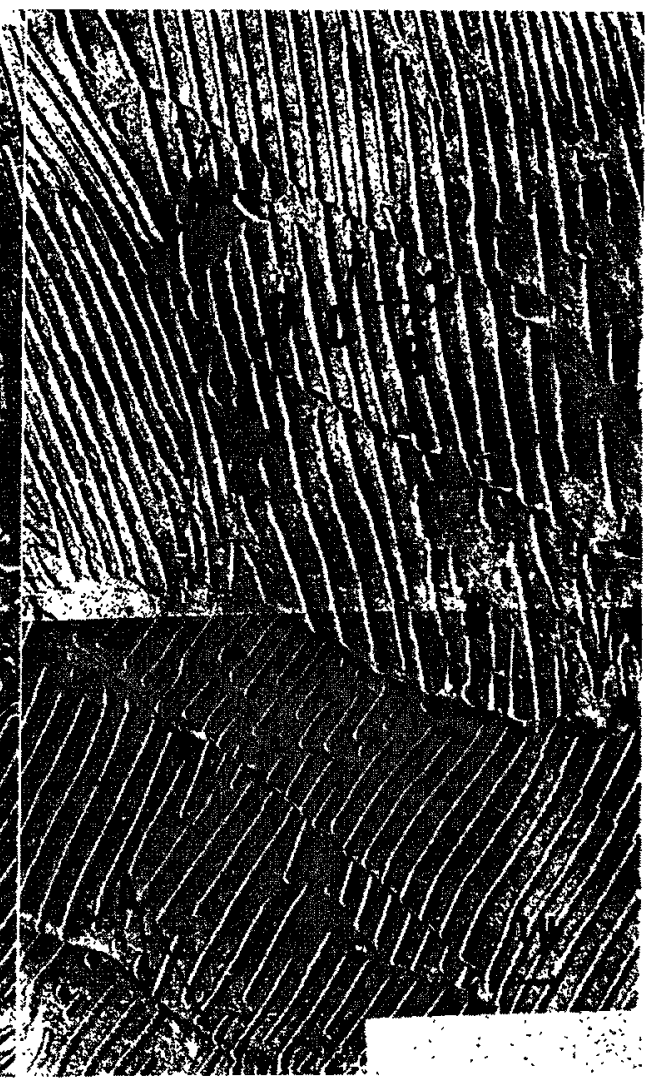

(b) After 7.8\% extension

Photo. 3 Two-stage replication electron microgrograph of the surface of a specimen before (a) and after (b) $7.8 \%$ extension

or that of slip propagation. Furthermore, the sites where shear of cementite occurs are occasionally apart from the intersection with slip bands (Photo. 2-D, Photo 3-D). The term "growth faults" was used by Puttick(10) for the deviations from the ideal structure of pearlite colonies of plane parallel lamellae. Actually, discontinuous steps are frequently found on the surface of cementites (Photo. 2-E, Photo. 3-C). Although it is not easy to reveal the fine details of shear in cementites, these steps, which presumably correspond to the growth faults, are considered to be related with the direction and the sites of shear. Further investigations now under way will be reported elsewhere.

\section{Discussions}

\section{Dependence of the flow stress on the pearlite morphology}

It is characteristic of the deformation of pearlite that there is a modified Hall-Petch relationship between the flow stress and mffp and that the Petch slope increases with strain. Embury and Fisher ${ }^{(7)}$ reported that the flow stress of heavily deformed pearlite increases with the subgrain size which is reduced with strain and with the initial interlamellar spacing. The dependence of the flow stress on the interlamellar spacing was formulated by a "modified" Hall-Petch relationship with the slope proportional to $\exp (\varepsilon / 4)$. However, for a small amount of deformation as in the case of this experiment, the dependence of the strain on the Petch slope can hardly be recognized because the factor $\exp (\varepsilon / 4)$ is almost unity.
As for the physical significance of the relation, the models generally accepted for the Hall-Petch relationship such as those proposed by Petch ${ }^{(22)}$ or Cottrell(23) are not likely to be applicable to the present case. One of the reasons is that the interlamellar spacings are too small to produce a sufficient length of the slip band whose stress concentration takes place at its end. Further the lamellar cementites are generally considered to be rigid barriers for transmitting slip into the neighboring ferrite.

Another theory for the interpretation of the Hall-Petch relation was proposed by $\mathrm{Li}^{(24)}$. He derived the same relation by calculating the internal stress due to the dislocations generated at grain boundaries, either by deformation of the boundary or pushing out of dislocations from sub-boundaries. This mechanism is very likely to operate but no evidence to support this theory has hitherto been observed in actual substances. On the other hand, it was revealed that the interface between cementite and ferrite can be a favorite nucleation site of dislocations $^{(18)}$. While this observation has never been related with the features of the flow stress of pearlite in either quantitative or qualitative way, this situation should be favorable for the model proposed by $\mathrm{Li}$.

The flow stress $\sigma$ in the stress field due to dislocations, the density of which is denoted by $\rho$, is given as ${ }^{(25)}$

(22) R. Armstrong, I. Codd, R. M. Douthwaite and N. J. Petch : Phil. Mag., 7 (1962), 45.

(23) A. H. Cottrell : Trans. Met. Soc. AIME, 212 (1958), 192.

(24) J. C. M. Li : Trans. Met. Soc. AIME, 227 (1963), 239.

(25) J.C.M. Li : Direct Obserwations of Imperfections in Crystals, Interscience, N..Y., (1952), p. 234. 


$$
\sigma=\sigma_{0}+\alpha \mu b \sqrt{\rho}
$$

where $\sigma_{0}$ and $\alpha$ are constants, $\mu$ shear modulus and $b$ the magnitude of the Burgers vector. The problem is then reduced to express $\rho$ as a function of the interlamellar spacing. In the following treatment, a deduction parallel to that by $\mathrm{Li}$ will be attempted.

Let us suppose as a simplified model what dislocations generated at the interface are distributed within the pearlitic ferrite. When the density of the dislocations generated in a unit area of the interface is denoted by $\delta\left(\mathrm{cm} / \mathrm{cm}^{2}\right)$, the increase in dislocation density, $\Delta \rho$, within the ferrite area is of the order of $\delta / l$, where $l$ is the average width of the pearlitic ferrite. It follows then that the strain produced is given by

$$
\Delta \varepsilon=b l \Delta \rho
$$

provided the average distance of the dislocation movement is taken as $l$.

The overall change of the dislocation density is partly due to the generation at the interface and partly to the multiplication and annihilation within the grain. These processes may be formulated as

$$
\frac{\mathrm{d} \rho}{\mathrm{d} \varepsilon}=\frac{1}{b l}
$$

and

$$
\frac{\mathrm{d} \rho}{\mathrm{d} \varepsilon}=\lambda \rho-\eta \rho^{2}
$$

respectively, where $\lambda$ and $\eta$ are constants. For a small amount of strain, the annihilation term may be neglected, but the multiplication term should be included to take into account the multiplication of dislocations generated at the interface between cementite and ferrite. A combination of eqs. (6) and (7) then gives the solution as

$$
\begin{aligned}
\rho & =\rho_{0} \exp (\lambda \varepsilon)+\frac{1}{\lambda b l}[\exp (\lambda \varepsilon)-1] \\
& \approx \rho_{0}(1+\lambda \varepsilon)+\varepsilon / b l .
\end{aligned}
$$

The last approximation is valid only for small strains. According to Keh and Weissman ${ }^{(26)}$, the dislocation density in a ferritic iron increases about ten times with the increase in strain from $1 \%$ to $10 \%$. Then the numerical value of $\lambda$ is estimated from eq. (7) to be of the order of twenty and the above approximation is justified for strains less than $5 \%$.

The initial density of dislocations, $\rho_{0}$, is to be of the order of $\delta / l$ in so far as the dislocations are supposed to have been generated at the interface. Then inserting eq. (9) into eq. (4) together with the approximation for $\rho_{0}$, the Hall-Petch relationship with the slope

$$
K=\alpha \mu b[\delta+(\lambda \delta+1 / b) \varepsilon]^{1 / 2}
$$

is obtained. The qualitative dependence of the Petch slope on the strain agrees fairly well with the experimentally observed one shown in Fig. 2. To proceed to a quantitative discussion, the value of $\delta$ is required, but its

(26) A.S. Keh and S. Weissmann: Electron Microscopy and Strength of Crystals, Interscience, N.Y., (1963), p. 231. exact estimation is rather difficult experimentally. It is assumed therefore that the contribution of the multiplication within the grains to the total dislocation density is negligible for small strains $(\sim 1 \%)$, and that the initial density of dislocations is $1 \times 10^{10} / \mathrm{cm}^{2}$. The former assumption may be reasonable for small strains in the substance in which the nucleation of fresh dislocations is easy, while the latter is rather arbitrary. The dislocation density of $1 \times 10^{10} / \mathrm{cm}^{2}$ is the value for iron with the grain size of 15 micron deformed several per cent at room temperature(26). Then it may be not a bad approximation for a eutectic steel which has fine lamellar spacings and shows the generation of dislocations at a rather early stage of deformation. Further, the resulting value of $\delta$ is not sensitive to the estimation of $K / K_{0}$.

The two assumptions stated above then give

$$
\delta=\rho_{0} \cdot l \simeq 1 \times 10^{6} / \mathrm{cm} \text {. }
$$

When the strain dependence of the Petch slope is assessed by the ratio of $K$ to its value at zero strain, $K_{0}$, i.e.

$$
K / K_{0}=(1+\varepsilon / b \delta)^{1 / 2},
$$

the calculated ratio at the strain of $5 \%$ using $1 \times 10^{6} / \mathrm{cm}$ for $\delta$ is 1.9 and the observed one is 1.3. This is considered to be a satisfactory agreement. It should be noted that the annihilation term in eq. (7) is neglected. The inclusion of the annihilation term reduces the calculated Petch slope at larger strains.

\section{Dependence of work-hardening on pearlite morphology}

The work-hardening rate $\mathrm{d} \sigma / \mathrm{d} \varepsilon$ of pearlite is directly formulated from eq. (4) and eq. (9) as

$$
\frac{\mathrm{d} \sigma}{\mathrm{d} \varepsilon}=\frac{\alpha \mu b}{2}\left[\rho_{0}(1+\lambda \varepsilon)+\varepsilon / b l\right]^{1 / 2}\left(\rho_{0} \lambda+\frac{1}{b l}\right) \text {. }
$$

This equation predicts a linear relationship between the work-hardening rate and the inverse square root of $\mathrm{mff}$, in good agreement with the experimental result shown in Fig. 3. The equation also indicates that the workhardening rate decreases approximately linearly with the inverse square root of strain. This is qualitatively consistent with the experimental results, but the observed dependence of work-hardening on the inverse square root of strain is not exactly linear. The absolute value of work-hardening rate calculated from eq. (13) is $500 \mathrm{~kg}$ / $\mathrm{mm}^{2}$ for the strain of $3 \%$ and the mffp of 1.1 micron, which is very close to the observed value.

\section{Dependence of the flow stress and work- hardening on the strain rate}

A characteristic of the deformation of pearlitic steel in contrast with that of ferritic iron is the minor dependence of the flow stress and work-hardening on the strain rate. In the case of ferritic iron, a long-range internal stress exerted by dislocation tangles and cell walls is considered to be strongly dependent on both the strain rate and temperature. It is a generally accepted idea that the formation of dislocation tangles and cell walls is due to double cross glides of screw dislocations ${ }^{(26)}$ and this 
mechanism also causes the multiplication of dislocations (27)(28).

In pearlitic steels, it has been reasoned that the flow stress is mainly composed of the long range internal stress due to the dislocations generated at the interface between cementite and ferrite. The observed dependence of the flow stress and work-hardening on pearlite morphology are best understood by the proposition that the dislocations newly generated at the interface have a larger effect on the internal stress than those multiplied within the pearlitic ferrite. Accordingly, the strain rate dependence of the flow stress will be mainly determined by the mechanism of the nucleation at the interface.

The nucleation mechanism like indentation or punching ${ }^{(29)}$ is very probable at the interface and this process is considered to be almost insensitive to the temperature as well as to the strain rate. The minor dependence of the flow stress or the work-hardening on the strain rate is then understood in the same way as in the case of its dependence on the pearlite morphology.

\section{The role of the cementite on the deformation of pearlite}

Throughout the above discussions, the features of the flow stress of pearlite are all ascribed to the generation of dislocations at the interface between cementite and ferrite. Concerning with the microscopic observations, the formation of slip within the pearlitic ferrite is considered to be an indication that the lamellar cementites are rather rigid barriers to the extension of the slip band although they eventually undergo shear at the intersection of slip bands. The analysis of the flow stress and workhardening also implies that the deformation of lamellar cementites are immaterial to the flow behavior of pearlite at small strains. The increase in internal stress, however, will result in the deformation of cracking of lamellar cementites. Since the deformation of a specimen cannot proceed without the deformation of cementites, the morphology or the structure of cementites may be effective to the ductility of the pearlitic steel.

(27) J. R. Low, Jr. and A. M. Turkalo : Acta Met., 10 (1962), 215.

(28) W. G. Johnston and J. J. Gilman : J. Appl. Phys, 31 (1960), 632.

(29) F. Seitz : Rev. Mod. Phys., 23 (1951), 328.

\section{Conclusions}

The mechanism which dominates the flow stress and work-hardening of pearlitic steel at small strains is investigated from both the analysis of the tensile properties and the microscopic observations of the slip patterns.

(1) The flow stress is found to increase linearly with the inverse square root of the mean free ferrite path, and the proportional constant increases with strain.

(2) The work-hardening rate, which is substantially large compared with that of ferritic iron, shows a similar dependence on the mean free ferrite path and the proportional constant decreases with strain.

(3) Fractional changes of both the flow stress and workhardening rate with the strain rate are less than those of ferritic iron.

(4) A large part of the flow stress is deduced to be due to the long range internal stress, and the effective stress which acts on dislocations is found to be almost the same as that in ferritic iron.

(5) By means of microscopic observations, the density of dislocations is observed to increase at the interface between cementite and ferrite, suggesting that the interface is an active nucleation sites of dislocations. The extension of slip band is shown to take place preferentially along the grain boundaries and through the gaps of lamellar cementites. Shear of cementites is eventually observed at the intersection with slip bands.

(6) The features of the deformation of pearlite stated above are consistently explained by the model in which dislocations, which are generated at the interface between cementite and ferrite rather than those multiplied within the grains, develop a large amount of long range internal stress within the pearlitic ferrite. Deformation of cementites is not likely to affect the flow stress or workhardening of pearlite.

\section{Acknowledgments}

The authors would like to thank Prof. S. Mizushima and Drs. T. Ohtake and K. Aoki for the encouragement throughout the course of the work. Their thanks are also due to Mr. S. Yamaguchi for the critical reading of the manuscript. 